\title{
Dexamethasone and sodium carboxymethyl cellulose prevent postoperative intraperitoneal adhesions in rats
}

\author{
X.H. Du ${ }^{1}$, J.Q. $\mathrm{Liu}^{2}, \mathrm{~K} . \mathrm{Xin}^{3}$ and G.H. Liu \\ ${ }^{1}$ Department of Hepatobiliary Surgery, Norman Bethune First Hospital, Jilin University, Jilin, China \\ ${ }^{2}$ Department of Radiation Medicine, School of Public Health, Jilin University, Jilin, China \\ ${ }^{3}$ Department of Emergency Surgery, Zhong Shan Hospital, Dalian University, Dalian, China \\ ${ }^{4}$ Department of Emergency Surgery, Norman Bethune First Hospital, Jilin University, Jilin, China
}

\begin{abstract}
We aimed to evaluate the effects of the barrier agent sodium carboxymethyl cellulose (SCMC) with and without dexamethasone for the prevention of postoperative adhesion formation in a rat model of postoperative peritoneal adhesion. A total of 160 threemonth old male and female Wistar rats underwent a laparotomy, and adhesions were induced by ileocecal abrasion. Rats were randomly assigned to 4 groups $(n=40$ each): group $A$, untreated; group B, treated with SCMC only; group C1, treated with SCMC $+3 \mathrm{mg}$ dexamethasone, and group $\mathrm{C} 2$, treated with SCMC $+8 \mathrm{mg}$ dexamethasone. After 12 days, adhesion formation and histopathological changes were compared. In groups A, B, C1, and C2, the mortality rates were $10,5,5$, and $5 \%$, respectively. In groups $\mathrm{C} 1$ and $\mathrm{C} 2$, the adhesions were filmy and easy to dissect and were milder compared with those in groups $\mathrm{A}$ and $\mathrm{B}$. The total adhesion score in group C1 $(3.38 \pm 0.49)$ was significantly lower than that of group B $(6.01 \pm 0.57 ; \mathrm{P}<0.01)$ or group $\mathrm{A}$ $(8.01 \pm 0.67 ; P<0.05)$. There was no significant difference in adhesion formation between groups $\mathrm{C} 1$ and $\mathrm{C} 2$. Compared with groups A and B, groups C1 and C2 exhibited milder histopathological changes. SCMC in combination with dexamethasone can prevent adhesion formation and is a better barrier agent than SCMC alone. The safety and feasibility of SCMC in combination with dexamethasone to prevent adhesion formation after abdominal surgery warrants further clinical study.
\end{abstract}

Key words: Dexamethasone; Sodium carboxymethyl cellulose; Adhesions

\section{Introduction}

Peritoneal adhesions, or bands of fibrous tissue connecting abdominal organs, are clinical complications caused by a wide variety of diseases and disorders. They are most common after abdominal surgery and are the leading cause of postoperative intestinal obstruction (1) and peritonitis (2). Attempts at preventing adhesions have included improving surgical techniques (3); reducing serosal injury; using anticoagulants such as heparin to prevent fibrin deposition $(4,5)$, hyaluronidase and streptokinase to remove fibrin exudation (6), or dexamethasone to inhibit fibroblastic growth; and applying sodium carboxymethyl cellulose (SCMC) (7). However, none of these methods effectively prevent peritoneal adhesions under all surgical conditions, and the incidence of postoperative abdominal adhesions is still very high (2).

Adhesion formation may be caused by abrasions between injured portions of the peritoneum, and the insertion of a barrier between segments of peritoneum can effectively prevent it (8). Thus, development of an effective, nontoxic, and bioreabsorbable material for adhesion prevention has become an important research goal (9-13). Local application of barrier agents such as SCMC has been shown to reduce the formation of adhesions in rats and humans, and it has been approved for clinical use $(14,15)$.

SCMC acts as an effective anti-adhesion agent by providing a mechanical barrier. A polysaccharide derivative, it is nontoxic, nonimmunogenic, nonpyrogenic, nonhemolytic, nonteratogenic, nonmutagenic, biodegradable, bioabsorbable, biocompatible $(16,17)$, and can be made into solutions and gels. Specifically, SCMC forms a protective membrane on the serosal surface, prevents the migration of cells including inflammatory cells, reduces fibroblast viability and proliferation, inhibits decreased fibrolytic activity, and prevents fibrin deposition on the injured peritoneum during peritoneal repair (18-21).

The glucocorticoid hormone dexamethasone strongly inhibits inflammation induced by a variety of physical, chemical, and immunological causes. In the early phases

Correspondence: Guohui Liu: <zhangxx063@sina.com>.

Received August 18, 2014. Accepted November 10, 2014. First published online February 24, 2015. 
of inflammation, dexamethasone can reduce exudation, edema, capillary dilation, leukocyte infiltration, and phagocytic reaction. In the late phases of inflammation, it can inhibit capillary proliferation and fibroblastic proliferation, reduce the formation of granulation tissues, and prevent adhesion and scar formation. To avoid the loss of dexamethasone in the wound due to accumulation of blood and body position, dexamethasone may be mixed with SCMC, which has a synergic effect on adhesion prevention.

\section{Material and Methods}

\section{Animals}

A total of 160 male and female Wistar rats (3 months old, $220 \pm 40 \mathrm{~g}$ ) were obtained from the animal center at the School of Medicine, Jinlin University, China. This study was carried out in strict accordance with the recommendations in the Guide for the Care and Use of Laboratory Animals of the National Institutes of Health. The protocol was approved by the Committee on the Ethics of Animal Experiments of the No. 1 Hospital of Jilin University, China [(2013) clinical trial (2013-121)]. All surgery was performed under sodium pentobarbital anesthesia, and all efforts were made to minimize suffering.

The rats were randomly assigned to 4 groups $(n=40$ each) according to treatment: group $A$, induction of adhesions with no further treatment; group $B$, induction of adhesions + SCMC; group C1, induction of adhesions + SCMC $+3 \mathrm{mg}$ dexamethasone; and group $\mathrm{C} 2$, induction of adhesions + SCMC + $8 \mathrm{mg}$ dexamethasone.

\section{Surgical technique}

We used the adhesion induction model previously described by Tarhan et al. (22). Rats were anesthetized with an intraperitoneal injection of $100 \mathrm{mg} / \mathrm{L}$ chloral hydrate $(250 \mathrm{mg} / \mathrm{kg}$ body weight; HeChang Company, China). A 4-cm midline incision was made in the abdomen, and the abdominal cavity was opened to expose the ileocecal junction. At the mesenteric border, the ileocecal junction was rubbed with dry sterile gauze to make a $1.0 \times 8.0 \mathrm{~cm}$ lesion. This was repeated approximately 10 times at the same area until hemorrhage points appeared. Care was taken to avoid damaging other tissues. For group A, no additional treatment was applied. For group $\mathrm{B}, 3 \mathrm{~mL}$ SCMC (provided by the Changchun Institute of Applied Chemistry, Chinese Academy of Science, China) was applied to the surface of the lesion. For groups $\mathrm{C} 1$ and $\mathrm{C} 2,3 \mathrm{~mL}$ SCMC with 3 or $8 \mathrm{mg}$ dexamethasone, respectively, was applied. The abdominal cavity was then closed. After surgery, rats were individually housed and fasted for $10 \mathrm{~h}$.

Twelve days after surgery, all rats were euthanized, and the abdominal adhesions were evaluated. A 2-cm ileocecal junction with adhesions was randomly selected, removed, and embedded in paraffin. The sections were examined under light microscope.

\section{Assessment of adhesions}

Abdominal adhesions were assessed by a surgeon blind to the experiments at 7-12 postoperative days, based on a scale described by Leach et al. (23). This scoring system takes into consideration abdominal adhesion range, extent, and intensity. Scores for the range of adhesions depended on the percent of the lesion area affected and the number of bands or location of adhesions: 0, no adhesion; 1, 1-25\% or one band; $2,26-50 \%$ or two bands; $3,51-75 \%$ or more than two bands, and $4,76-100 \%$ or direct adhesions to the abdominal wall. The extent of adhesions was scored $0,1,2$, or 3 for no adhesions, filmy thickness, dense thickness, and dense thickness with vascularity, respectively. The intensity of adhesions was scored based on the ease of dissection: 0 for no adhesions, 1 for easy tears, 2 for blunt dissection, and 3 for sharp dissection. The final adhesion scores were the sum of the scores for range, extent, and intensity. The maximum score possible was 10 .

\section{Statistical analyses}

Statistical analyses were performed using the SAS 9.0 software (USA). Values are reported as means \pm SD. Student's $t$-tests were performed to identify significant differences between two independent groups. Probability values $<0.05$ were considered to be statistically significant.

\section{Results}

\section{Postoperative deaths and wound healing}

Four rats in group $A$, in which adhesions were experimentally induced and untreated, died 5-8 days after surgery. Two rats in group $\mathrm{B}$, in which adhesions were induced and then treated with $3 \mathrm{~mL}$ SCMC only, died 8 days after surgery. Rats in groups $\mathrm{C} 1$ and $\mathrm{C} 2$ were induced to develop adhesions and treated with $3 \mathrm{~mL}$ SCMC mixed with either 3 or $8 \mathrm{mg}$ dexamethasone, respectively. Two rats in each group died 3-4 days after surgery. Thus, the mortality rates were $10,5,5$, and $5 \%$ in groups $A, B, C 1$, and $C 2$, respectively. Autopsies showed that 3 rats in group $A$ and 2 rats in group $B$ died of strangulation necrosis induced by severe bowel adhesions. These rats were assigned total adhesion scores of 10 . One rat in group A, 2 rats in group $\mathrm{C} 1$, and 2 rats in group $\mathrm{C} 2$ died of traumatic shock induced by striped ileum perforation. No adhesions were observed in the rats that died of traumatic shock, and these animals were excluded from the study.

The wound healing rate was $100 \%$ for groups $A, B$, and C1, and $90 \%$ for group C2. In group C2, wound healing was delayed in 4 rats.

\section{Evaluation of adhesions}

In group A, the adhesions were extensive and dense and vascularized in some of the rats. Blunt or sharp dissection was usually required to separate adhesions. In groups C1 and $\mathrm{C} 2$, the adhesions were mild and filmy, and were easily dissected. The adhesions in group B were more severe than 
Table 1. Adhesion scores of rats of each group.

\begin{tabular}{lccccc}
\hline Group & $\mathrm{n}$ & \multicolumn{3}{c}{ Adhesion scores } \\
\cline { 3 - 6 } & & Range & Extent & Intensity & Total \\
\hline A & 49 & $3.07 \pm 0.89$ & $2.43 \pm 0.52$ & $2.51 \pm 0.45$ & $8.01 \pm 0.67$ \\
B & 50 & $2.29 \pm 0.60$ & $1.75 \pm 0.45$ & $1.97 \pm 0.45$ & $6.01 \pm 0.57$ \\
C1 & 47 & $1.13 \pm 0.32$ & $1.03 \pm 0.29$ & $1.22 \pm 0.28$ & $3.38 \pm 0.49^{*}$ \\
C2 & 50 & $1.17 \pm 0.33$ & $1.02 \pm 0.27$ & $1.21 \pm 0.26$ & $3.40 \pm 0.50$ \\
\hline
\end{tabular}

Group A, untreated; group B, treated with sodium carboxymethyl cellulose (SCMC) only; group C1, treated with SCMC $+3 \mathrm{mg}$ dexamethasone, and group $\mathrm{C} 2$, treated with $\mathrm{SCMC}+8 \mathrm{mg}$ dexamethasone. ${ }^{*} \mathrm{P}<0.05$, group $\mathrm{C} 1$ compared with group $\mathrm{B}$; ${ }^{*} \mathrm{P}<0.01$, group C1 compared with group A; $\mathrm{P}>0.05$, group C1 compared with group C2 (Student's $t$-test).

those in group $\mathrm{A}$, but milder than those in groups $\mathrm{C} 1$ and $\mathrm{C} 2$. The mean adhesion score in group $\mathrm{C} 1(3.38 \pm 0.49)$ was significantly lower than that of group $B(6.01 \pm 0.57, P<0.01)$ and group $\mathrm{A}(8.01 \pm 0.67, \mathrm{P}<0.05$; Table 1). There was no significant difference in adhesion formation between groups $\mathrm{C} 1$ and $\mathrm{C} 2(\mathrm{P}>0.05)$.

\section{Histopathological findings}

In group $A$, the dissected surface of the serosa might be unrepaired with discontinuous mesothelial cells (Figure 1). Capillary and fibroblastic proliferation, dense collagen fibers, and infiltration of large numbers of granulocytes and macrophages were observed. Compared with group $A$, group $B$ exhibited milder histopathological changes (Figure 2). The serosa had a partially repaired dissection surface with broken mesothelial cells. Capillary and fibroblastic proliferation, dense collagen fibers, and granulocyte and macrophage infiltration were still observed. In groups C1 and $\mathrm{C} 2$, the serosa had a repaired dissection surface with continuous mesothelial cells, and no obvious fibroblastic proliferation was found (Figure 3). A little capillary proliferation, loose collagen fibers, and a small number of scattered granulocytes and macrophages were observed.

\section{Discussion}

We have developed a gel-like bioabsorbable SCMC in the form of a gel-like liquid with high fluidity that uniformly distributes in each part of the abdominal cavity, thus producing complete and extensive separation between peritonea. We found that $3 \mathrm{mg}$ dexamethasone thoroughly mixed with SCMC produced an excellent anti-adhesion effect in a rat model of peritoneal adhesion. Dexamethasone in combination with SCMC significantly decreased the range, extent, and intensity of adhesions compared with SCMC alone. However, we did not find any difference in adhesion formation between groups $\mathrm{C} 1$ (3 $\mathrm{mg}$ dexamethasone + SCMC) and C2 (8 mg dexamethasone + SCMC), suggesting that the higher dose of dexamethasone did not prevent adhesion formation to a greater degree than the lower dose. In addition, 4 rats in group C2 exhibited delayed wound healing that was not evident in any of the other groups, implying that $8 \mathrm{mg}$ dexamethasone may delay wound healing. Therefore, of the two doses tested in this study, $3 \mathrm{mg}$ is more efficacious in preventing adhesion formation and avoiding potential side effects.

It was previously reported that adhesions in this model

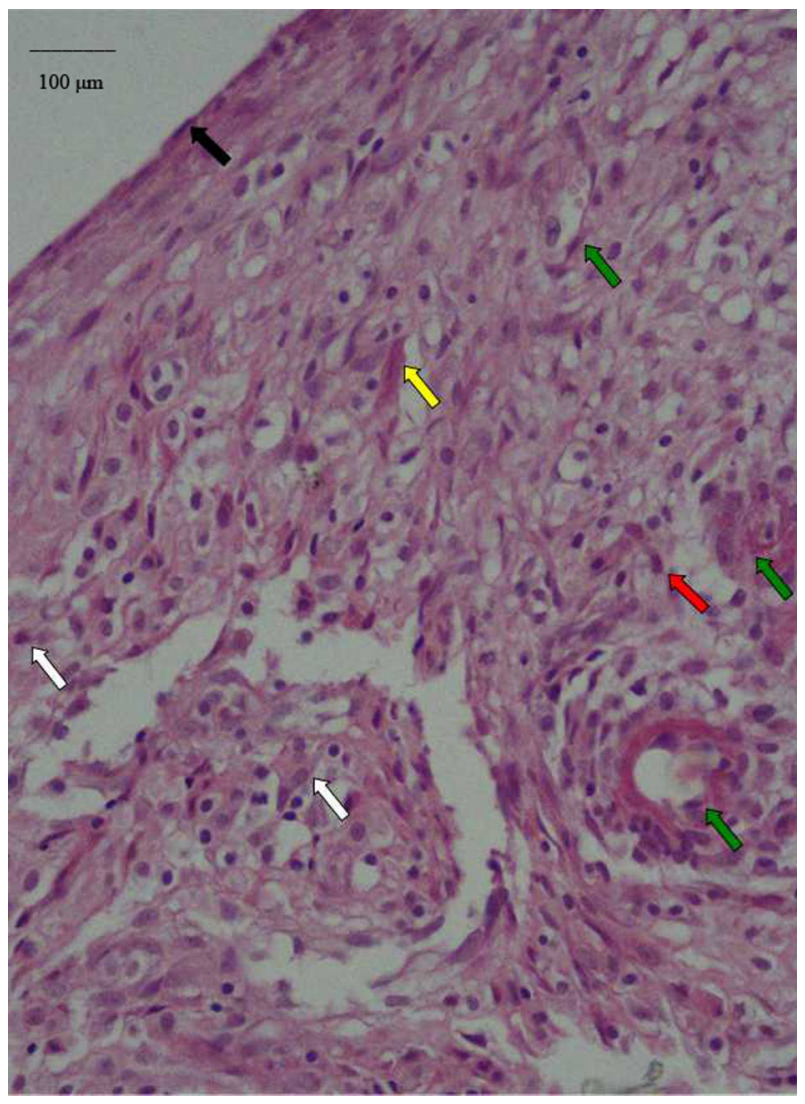

Figure 1. Hematoxylin-eosin staining of the serosa in a representative rat in group $\mathrm{A}$ (untreated). The serosa has an unrepaired dissection surface with discontinuous mesothelial cells (black arrow). Capillary proliferation (green arrow), fibroblastic proliferation (red arrow), dense collagen fibers (yellow arrow), and infiltration of a large number of granulocytes and macrophages (white arrows) were observed $(100 \times)$ 


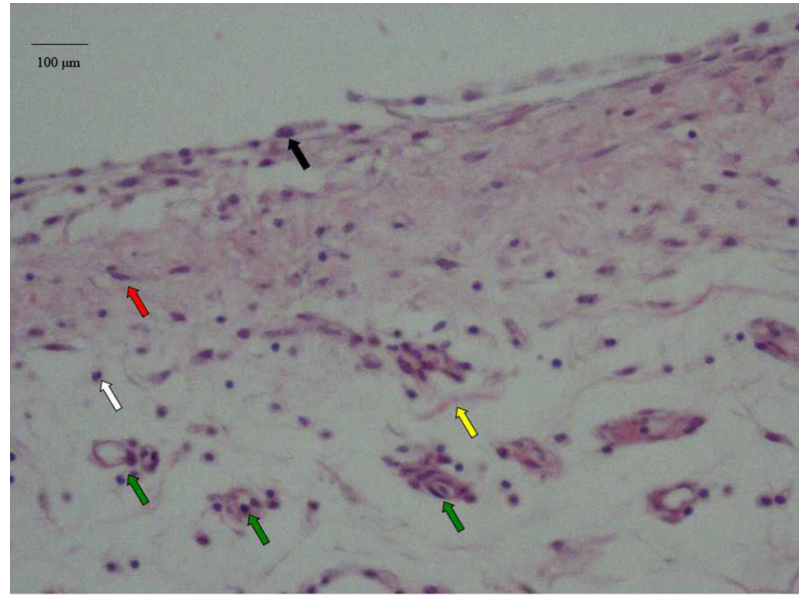

Figure 2. Hematoxylin-eosin staining of the serosa in a representative rat in group $\mathrm{B}$ (treated with sodium carboxymethyl cellulose [SCMC] only). The serosa has a partially repaired dissection surface with broken mesothelial cells (black arrow). Capillary proliferation (green arrows), fibroblastic proliferation (red arrow), dense collagen fibers (yellow arrow), and infiltration of granulocytes and macrophages (white arrow) were observed $(100 \times)$.

were easily separated at postoperative days 1-3 but more difficult to dissect at postoperative day 7 (24). Intestinal dilation, edema, and congestion were observed in the intestine proximal to adhesions, while the intestine distal to adhesions was normal. Electron microscopy inspection showed that at postoperative day 1 , when adhesions began to form, the adhesion tissues mainly consisted of fibrin exuded in the acute phase of inflammation and a large number of neutrophils (G.H. Liu, unpublished data). Lymphocytes, plasma cells, and macrophages were rarely found. In the present study, we found that at postoperative days $3-5$, neutrophils gradually decreased, accompanied by an increase in macrophages. Fibroblast proliferation and differentiation were observed. At postoperative days 5-7, the meshwork originally formed by fibrin was replaced by bundles of coarse collagen fibers. The numbers of macrophages and fibroblasts increased many-fold and were arranged parallel with the collagen fibers. Thus, the most crucial time for adhesion formation was at postoperative day 7 . If the inserted barrier can last for 1 week, the damaged peritoneum will regenerate to prevent adhesion formation. However, in the present study, SCMC without dexamethasone was degraded and completely reabsorbed by postoperative day 5 (G.H. Liu, unpublished data), resulting in poor prevention of adhesion formation.

Combining SCMC and dexamethasone utilizes the advantages of both to reduce adhesion formation, including dexamethasone's pharmacological effects in reducing inflammatory exudation and inhibiting capillary and fibroblast proliferation. In this study, we mixed dexamethasone with a

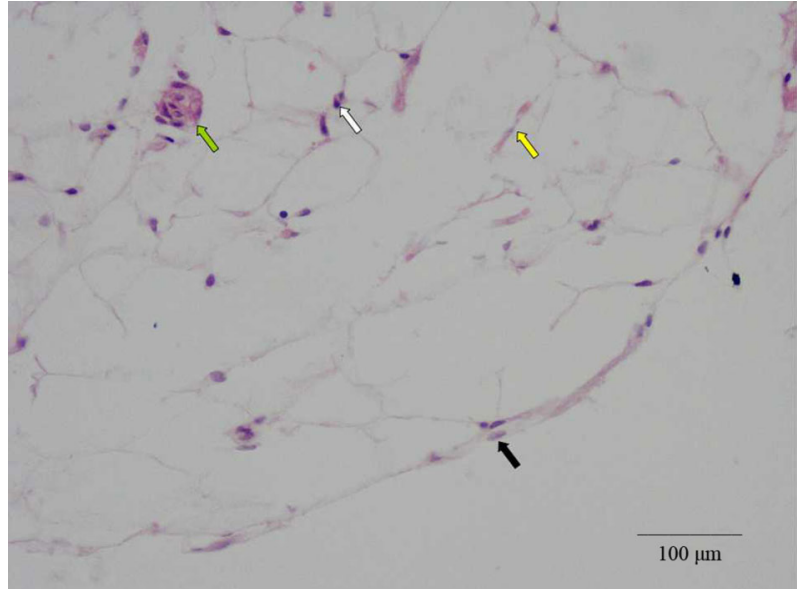

Figure 3. Hematoxylin-eosin staining of the serosa in a representative rat in group $\mathrm{C} 1$ (treated with sodium carboxymethyl cellulose [SCMC] $+3 \mathrm{mg}$ dexamethasone). The serosa has a repaired dissection surface with continuous mesothelial cells (black arrow). No obvious fibroblastic proliferation was found. Capillary proliferation (green arrow), loose collagen fibers (yellow arrow), and a small number of scattered granulocytes and macrophages (white arrow) were observed $(100 \times)$.

pre-prepared gel-like SCMC liquid. This allowed the mixture to become uniformly distributed throughout the abdominal cavity due to the bowel's movement and produced a complete and extensive separation between peritonea. In the present study at postoperative day 3, laparotomy examination revealed a small amount (approximately $2 \mathrm{~mL}$ ) of exudate in the abdominal cavity. At postoperative day 7 , the exudate was absorbed, and a transparent gel-like SCMC membrane (approximately $1 \mathrm{~cm}$ thick) was formed on the surface of the lesions between the abdominal wall and the intestine, and between the intestines. At postoperative day 9, there were some residual transparent gellike SCMC membranes on the lesion surfaces. At postoperative day 12 , the injured serosae were almost fully repaired. Loose adhesions were occasionally observed on the surface of lesions between the abdominal wall and the intestine. No intestinal dilations, edema, or congestion were found, and the color of the intestine was normal. Therefore, the degradation and complete reabsorption of SCMC required more than 7 days.

In conclusion, SCMC in combination with dexamethasone can prevent adhesion formation, inhibit migration of cells including inflammatory cells, reduce fibroblast viability and proliferation, prevent fibrin deposition on the serosal surface, and form a protective membrane on the serosal surface. SCMC in combination with dexamethasone does not irritate the mucosa or affect bowel movements. The safety and feasibility of SCMC in combination with dexamethasone to prevent adhesion formation after abdominal surgery warrants further clinical study. 


\section{Acknowledgment}

We thank Medjaden Bioscience Limited for assisting in the preparation of this manuscript.

\section{References}

1. Harris DA, Topley N. Peritoneal adhesions. Br J Surg 2008; 95: 271-272.

2. Wilson MS. Practicalities and costs of adhesions. Colorectal Dis 2007; 9 (Suppl 2): 60-65, doi: 10.1111/j.1463-1318.2007. 01360.x.

3. Parsak CK, Satar S, Akcam T, Satar D, Sungur I. Effectiveness of treatment to prevent adhesions after abdominal surgery: an experimental evaluation in rats. Adv Ther 2007; 24: 796-802, doi: $10.1007 / B F 02849972$.

4. Williams SA, Schwarzbauer JE. A shared mechanism of adhesion modulation for tenascin- $\mathrm{C}$ and fibulin-1. Mol Biol Cell 2009; 20: 1141-1149, doi: 10.1091/mbc.E08-06-0621.

5. Hirsh J, Bauer KA, Donati MB, Gould M, Samama MM, Weitz JI. Parenteral anticoagulants: American College of Chest Physicians Evidence-Based Clinical Practice Guidelines (8th Edition). Chest 2008; 133: 141S-159S, doi: 10.1378/chest. 08-0689.

6. Wang P, Wang J, Zhang W, Li Y, Li J. Effect of the combination of fibrin glue and growth hormone on intestinal anastomoses in a pig model of traumatic shock associated with peritonitis. World J Surg 2009; 33: 567-576.

7. Bae JS, Jin HK, Jang KH. The effect of polysaccharides and carboxymethylcellulose combination to prevent intraperitoneal adhesion and abscess formation in a rat peritonitis model. J Vet Med Sci 2004; 66: 1205-1211.

8. DeCherney AH, diZerega GS. Clinical problem of intraperitoneal postsurgical adhesion formation following general surgery and the use of adhesion prevention barriers. Surg Clin North Am 1997; 77: 671-688, doi: 10.1016/S0039-6109 (05)70574-0

9. Fundueanu G, Constantin M, Dalpiaz A, Bortolotti F, Cortesi R, Ascenzi $P$, et al. Preparation and characterization of starch/ cyclodextrin bioadhesive microspheres as platform for nasal administration of Gabexate Mesylate (Foy) in allergic rhinitis treatment. Biomaterials 2004; 25: 159-170, doi: 10.1016/ S0142-9612(03)00477-0.

10. diZerega GS, Cortese S, Rodgers KE, Block KM, Falcone SJ, Juarez TG, et al. A modern biomaterial for adhesion prevention. J Biomed Mater Res B Appl Biomater 2007; 81: 239-250, doi: 10.1002/jbm.b.30659.

11. Darmas B. Use of barrier products in the prevention of adhesion formation following surgery. $J$ Wound Care 2008; 17: 405-8, 411, doi: 10.12968/jowc.2008.17.9.30939.

12. Ahmad G, Duffy JM, Farquhar C, Vail A, Vandekerckhove P, Watson A, et al. Barrier agents for adhesion prevention after gynaecological surgery. Cochrane Database Syst Rev 2008; CD000475.

13. Mahdy T, Mohamed G, Elhawary A. Effect of methylene blue on intra-abdominal adhesion formation in rats. Int $J$ Surg
2008; 6: 452-455.

14. Dilege E, Coskun H, Gunduz B, Sakiz D, Mihmanli M. Prevention of adhesion to prosthetic mesh in incisional ventral hernias: comparison of different barriers in an experimental model. Eur Surg Res 2006; 38: 358-364, doi: 10.1159/ 000094382.

15. Wallwiener M, Brucker S, Hierlemann H, Brochhausen C, Solomayer $\mathrm{E}$, Wallwiener $\mathrm{C}$. Innovative barriers for peritoneal adhesion prevention: liquid or solid? A rat uterine horn model. Fertil Steril 2006; 86: 1266-1276, doi: 10.1016/j.fertnstert. 2006.05.023.

16. Adanali G, Verdi M, Tuncel A, Erdogan B, Kargi E. Effects of hyaluronic acid-carboxymethylcellulose membrane on extraneural adhesion formation and peripheral nerve regeneration. J Reconstr Microsurg 2003; 19: 29-36, doi: 10.1055/ s-2003-37188.

17. Corrales F, Corrales M, Schirmer CC. Preventing intraperitoneal adhesions with vitamin $\mathrm{E}$ and sodium hyaluronate/carboxymethylcellulose: a comparative study in rats. Acta Cir Bras 2008; 23: 36-41, doi: 10.1590/S0102-86502008000100007.

18. Sousa AA, Petroianu A, Trapiello Neto V, Rios VS, Barbosa AJ. Effect of sodium carboxymethylcellulose and methylprednisolone on the healing of jejunal anastomoses in rats. Braz J Med Biol Res 2001; 34: 519-523, doi: 10.1590/ S0100-879X2001000400012.

19. Eggleston RB, Mueller PO, Parviainen AK, Groover ES. Effect of carboxymethylcellulose and hyaluronate solutions on jejunal healing in horses. Am J Vet Res 2004; 65: 637-643.

20. Ogunlewe JO, Osegbe DN. Zinc and cadmium concentrations in indigenous blacks with normal, hypertrophic, and malignant prostate. Cancer 1989; 63: 1388-1392, doi: 10.1002/10970142(19890401)63:7<1388::AID-CNCR2820630725>3.0.CO; 2-M.

21. Isaji M, Naito J. Comparative studies on inflammatory reactions induced by non-immunological and immunological stimuli in an air pouch and in a carboxymethyl cellulose (CMC)-induced inflammatory pouch. Int J Exp Pathol 1992; 73: 231-239.

22. Tarhan OR, Barut I, Sezik M. An evaluation of normal saline and taurolidine on intra-abdominal adhesion formation and peritoneal fibrinolysis. J Surg Res 2008; 144: 151-157, doi: 10.1016/j.jss.2007.09.006.

23. Leach RE, Burns JW, Dawe EJ, SmithBarbour MD, Diamond MP. Reduction of postsurgical adhesion formation in the rabbit uterine horn model with use of hyaluronate/carboxymethylcellulose gel. Fertil Steril 1998; 69: 415-418, doi: 10.1016/S00150282(97)00573-6.

24. Du X, Hong G, Sun P, Liu G. $Z^{2+}-S C M C$ versus HA for preventing intraperitoneal adhesions: a rat model study. Int $\mathrm{J}$ Med Sci 2012; 9: 467-471. 